\title{
Lack of association between expression of MRP2 and early relapse of colorectal cancer in patients receiving FOLFOX-4 chemotherapy
}

\author{
MOJGAN MIRAKHORLI $^{1,6}$, NASRIN SHAYANFAR ${ }^{2}$, SABARIAH ABDUL RAHMAN $^{1,3}$, \\ ROZITA ROSLI $^{4}$, SYAHRILNIZAM ABDULLAH ${ }^{4}$ and AHAD KHOSHZABAN ${ }^{5-7}$
}

\begin{abstract}
${ }^{1}$ Pathology Department, Faculty of Medicine and Health Sciences, University Putra Malaysia, 43400 UPM Serdang, Malaysia;
${ }^{2}$ Pathology Department, Hazrate Rasoul Akram Hospital, Tehran University, Tehran, Iran;

${ }^{3}$ Cluster of Medical Laboratory Sciences, Faculty of Medicine, Universiti of Teknologi MARA, Selayang Campus, 68100,

Batu Caves, Selangor, Malaysia; ${ }^{4}$ Medical Genetics Laboratory, Faculty of Medicine and Health Sciences,

University Putra Malaysia, 43400 UPM Serdang, Selangor, Malaysia; ${ }^{5}$ Iranian Tissue Bank Research and Preparation Center, Imam Khomeini Hospital Complex; ${ }^{6}$ Stem Cell Preparation Unit, Eye Research Center, Farabi Eye Hospital;

${ }^{7}$ Dental Bio Material Department, Faculty of Dentistry, Tehran University of Medical Science, Tehran, Iran
\end{abstract}

Received February 25, 2012; Accepted June 21, 2012

DOI: $10.3892 / \mathrm{ol} .2012 .889$

\begin{abstract}
Recurrence following failure of chemotherapy limits the application of high doses of anticancer drugs currently used for eliminating cancerous cells. It has been identified that ATP-binding cassette (ABC) multidrug transporters are associated with chemoresistance, which is a major obstacle in cancer therapy. The present study aimed to investigate the association of pretherapeutic multidrug resistance-associated protein 2 (MRP2) expression with response to chemotherapy in stage II/III colorectal cancer (CRC). Protein expression was determined by immunohistochemical analysis of 50 archival samples from patients who had not received preoperative chemotherapy and radiotherapy. All patients were treated with 5-fluorouracil/leucovorin (FL) plus oxaliplatin (FOLFOX-4) regimen for 6 months following curative resection. During the 12 months of follow-up, local and distant recurrences were observed in $15(30 \%)$ cases, of which 5 occurred at the time of chemotherapy. MRP2 expression was observed in 24 (48\%) and $7(14 \%)$ cases in the tumor tissues and matched normal tissues,
\end{abstract}

Correspondence to: Professor Sabariah Abdul Rahman, Cluster of Medical Laboratory Sciences, Faculty of Medicine, Universiti of Teknologi MARA, Selayang Campus, 68100, Batu Caves, Selangor, Malaysia

E-mail: srahman0550@yahoo.com

Dr Ahad Khoshzaban, Department of Dental Biomaterials, Dental School, Tehran University of Medical Sciences, Tehran, Iran E-mail: akhoshzaban@gmail.com

Key words: multiple drug resistance protein 2, FOLFOX-4, colorectal cancer, relapse respectively. A significant difference was observed between the positive expression frequency in the tumor tissues compared to the surrounding normal mucosa $(\mathrm{P}=0.003)$. The incidence of recurrence and metastasis for patients in the MRP2-positive group was lower than that in the MRP2-negative group $(\mathrm{P}>0.05)$; however, all 5 cases who demonstrated recurrence during their treatment were MRP2-positive $(\mathrm{P}=0.022)$. MRP2 expression was not correlated with the clinicopathological markers in this group of patients. Kaplan-Meier analysis revealed that MRP2 expression was not associated with a shorter disease-free survival or overall survival of patients $(\mathrm{P}>0.05)$. The results of this study indicated that MRP2 is overexpressed in the course of CRC development and progression. However, expression of MRP2 was not associated with recurrence of patients treated with FL and oxaliplatin in the population studied.

\section{Introduction}

High incidence of mortality from colorectal cancer (CRC) in Western countries has prompted much research on CRC within these countries. In Iran, there has been a significant increase in CRC cases, particularly among the younger population (1). According to a previous study, there are an estimated 3,641 new cases of CRC in Iran each year, of which 2,262 (62\%) result in mortality (2). Therefore, it is necessary to draw attention to $\mathrm{CRC}$ research in order to improve the diagnosis and treatment of this type of cancer. Adjuvant chemotherapy following curative resection is the most common approach to CRC treatment; however, a decreased sensitivity to chemotherapeutic agents has been observed in CRC patients who were subjected to previous surgery (3).

Overexpression of ATP-binding cassette (ABC) transporter proteins was established to confer drug resistance to tumor cells (4). Multiple drug resistance protein 2 (MRP2/ABCC2), a member of the ABC multidrug transporters, is a polytopic 
membrane glycoprotein of 190-200 kDa, which has two ATP-binding domains and 17 transmembrane regions (5), and was previously named the canalicular multispecific organic anion transporter (cMOAT). MRP2 is present in the apical membrane of polarized cells within the liver, kidney and intestine (6) and plays an important role in in vitro studies into drug resistance $(7,8)$. It exports a wide spectrum of substrates using an ATP-dependent mechanism, including the glucuronide, glutathione and sulfate conjugates of endogenous and exogenous compounds $(9,10)$.

Glutathione conjugation was identified as one of the mechanisms for oxaliplatin resistance in CRC (11). The FOLFOX-4 regimen is the main chemotherapeutic procedure used to treat $\mathrm{CRC}$; it is a combination of oxaliplatin (a third-generation platinum drug) and 5-fluorouracil/leucovorin (FL). Incorporation of oxalipatin into a backbone of FL is able to improve the rate of response by $40-50 \%$ in metastatic CRC cases (12). Several mechanisms contribute to resistance against platinum compounds, including enhanced DNA repair, decreased drug accumulation, drug inactivation and enhanced tolerance to platinum-DNA adducts $(13,14)$. Glutathione conjugation is a well-known mechanism involved in the detoxification and inactivation of platinum compounds (15).

The role of the MRP2 gene has also been identified in cisplatin resistance (16). The functional inhibition of MRP2 appears to be an effective approach in overcoming resistance to platinum-based drugs in human melanoma cells (17). A recent in vivo study demonstrated the involvement of MRP2 in drug resistant phenotypes of CRC cell lines (18). However, the role of MRP2 in the clinical outcome of CRC patients who received platinum-based therapy remains to be clarified.

In this hospital-based study, we performed immunohistochemical detection of MRP2 in paraffin-embedded samples of 50 CRC patients. We investigated the putative association of MRP2-positivity and early CRC relapse in patients who were treated with FL and oxaliplatin.

\section{Patients and methods}

Study population and chemotherapy. A total of $50 \mathrm{CRC}$ patients (30 males and 20 females; age range, 17-77 years) who had undergone complete resection of histologically verified stage II (T2 and T3, N0, M0) or stage III (any T, N1 and 2, M0) CRC were selected for this study. The clinical stage and pathological features of primary tumors were defined according to the criteria of the American Joint Commission on Cancer/International Union against Cancer (AJCC/UICC) (19). This study examined protein expression in association with platinum-based drugs; therefore, patients who had received prior chemotherapy or radiotherapy were excluded, thus only the patient's first response to chemotherapy was analyzed. The clinicopathological features of the patients were obtained from their medical records. This study was approved by the Hazrate Rasoul Akram Hospital (Tehran University, Tehran, Iran) and the Faculty of Medicine and Health Sciences (University Putra Malaysia, Malaysia).

All patients were treated with 12 cycles of FOLFOX-4 chemotherapy for 6 months. The chemotherapeutic regimen consisted of oxaliplatin $\left(85 \mathrm{mg} / \mathrm{m}^{2}\right)$ combined with leucovorin $\left(200 \mathrm{mg} / \mathrm{m}^{2}\right)$ and bolus fluorouracil $\left(400 \mathrm{mg} / \mathrm{m}^{2}\right)$ on day 1 , and continuous infusion of fluorouracil $\left(600 \mathrm{mg} / \mathrm{m}^{2}\right)$ on day 2 . The cycle was then repeated after a 2 -week rest period. Patients received premedication, including dexamethasone or granisetron, $1 \mathrm{~h}$ prior to their treatment. Clinical response was assessed by measuring carcinoembryonic antigen (CEA) levels at 3-month intervals for 2 years and at 6-month intervals thereafter. A colonoscopy and CT scan was usually performed at 6-month intervals in the first 2 years and annually thereafter; these tests were mandatory following an elevated CEA level. Development of new recurrent or metastatic lesions following surgery was considered as relapse and local relapse was histopathologically/cytologically confirmed by specimen examination.

Immunohistochemistry. The tumor and matched normal paraffin-embedded tissues were cut into $4 \mu \mathrm{m}$ sections, mounted onto slides and incubated for $30 \mathrm{~min}$ at $60^{\circ} \mathrm{C}$. Sections were routinely deparaffinized in xylene and rehydrated in a graded ethanol series. Endogenous peroxidase activity was blocked by incubation in $0.3 \% \mathrm{H}_{2} \mathrm{O}_{2}$ for $30 \mathrm{~min}$. Antigen retrieval was achieved by heating the tissue sections in an autoclave filled with sodium citrate buffer for $10 \mathrm{~min}$ at $120^{\circ} \mathrm{C}$. Following cooling at room temperature, the sections were rinsed in phosphate-buffered saline (PBS; pH 7.4), incubated with anti-MRP2 primary antibodies (M2III-6, ab3373; Abcam, Cambridge, UK) diluted at 1:120 for $1.5 \mathrm{~h}$, and rinsed in PBS. The sections were subsequently treated with EnVision+ Dual Link System-HRP secondary antibody (K4061; Dako, Copenhagen, Denmark) for $45 \mathrm{~min}$ and rinsed in PBS. Immunoreactivity was visualized with 3,3'-diaminobenzidine (Dako). The sections were counterstained with hematoxylin and mounted. The protein expression was evaluated by two pathologists, blinded to clinical outcomes. Liver tissues were used as a positive control for MRP2 (Fig. 1C) and the primary antibody was replaced by PBS as a negative control. Positive and negative controls were applied in each run of the procedure. Samples were classified as positive for the expression when over $10 \%$ of tumor cells demonstrated immunoreactivity, as in previous studies in ovarian cancer by Arts et al (20) and in small cell lung cancer by Ushijima et al (21).

Statistical analysis. The data were analyzed using the Statistical Package for the Social Sciences software version 11.0 (SPSS, Inc., Chicago, IL, USA). Correlations between positive expression, clinicopathological features and early relapse were analyzed using a Chi-square test. The Kaplan-Meier method was used for survival analyses and the log-rank test was used for comparing survival data. Disease-free survival (DFS) was defined as the number of months from the date of surgery to the first event of documented relapse or mortality. For those patients who did not experience relapse, data were collected during the last follow-up. Overall survival (OS) was considered as the duration between surgery and mortality from any cause, and data on survivors were counted at the last follow-up. $\mathrm{P}<0.05$ was considered to indicate a statistically significant difference.

\section{Results}

Patient characteristics. In total, 50 patients were included in this study, of which 30 were male and 20 were female (male 
Table I. Clinicopathological data of patients and tumors.

\begin{tabular}{|c|c|c|}
\hline Characteristics & $\begin{array}{l}\text { No. of } \\
\text { cases }\end{array}$ & $\%$ \\
\hline \multicolumn{3}{|l|}{ Gender } \\
\hline Male & 30 & 60 \\
\hline Female & 20 & 40 \\
\hline \multicolumn{3}{|l|}{ Age } \\
\hline$>50$ & 38 & 76 \\
\hline$<50$ & 12 & 24 \\
\hline Mean (years) & 57.2 & \\
\hline Median (years) & 57 & \\
\hline Range (years) & $17-77$ & \\
\hline \multicolumn{3}{|l|}{ Tumor size (cm) } \\
\hline$<5$ & 28 & 56 \\
\hline$>5$ & 22 & 44 \\
\hline \multicolumn{3}{|l|}{ Location } \\
\hline Colon & 35 & 70 \\
\hline Rectum & 15 & 30 \\
\hline \multicolumn{3}{|c|}{ Depth of tumor invasion } \\
\hline $\mathrm{T} 4$ & 3 & 6 \\
\hline $\mathrm{T} 3$ & 47 & 94 \\
\hline \multicolumn{3}{|c|}{ Lymph node metastasis } \\
\hline Negative & 22 & 44 \\
\hline Positive & 28 & 56 \\
\hline \multicolumn{3}{|l|}{ Stage } \\
\hline II & 22 & 44 \\
\hline III & 28 & 56 \\
\hline \multicolumn{3}{|l|}{ Histology } \\
\hline $\mathrm{WD}+\mathrm{MD}$ & 48 & 96 \\
\hline $\mathrm{PD}$ & 2 & 4 \\
\hline
\end{tabular}

WD, well-differentiated; MD, moderately differentiated; PD, poorly differentiated.

to female ratio, 1.5 to 1$)$. A total of 38 patients (76\%) were $>50$ years and $12(24 \%)$ were $<50$ years (range, $17-77$ years). A total of 22 cases $(44 \%)$ were stage II and 28 cases $(56 \%)$ were stage III. The primary tumor location for $35(70 \%)$ patients was the colon and for $15(30 \%)$ was the rectum. Table I shows the clinicopathological features of the patients and tumors. The median follow-up time was 34 months (range, 24-50 months); in this time, a total of 15 cases (30\%) developed early relapse, either local recurrence or distant metastasis, of which 5 were strongly resistant to treatment and demonstrated recurrence at time of chemotherapy.

Protein expression. Expression and subcellular localization of MRP2 was determined by immunohistochemistry of 50 paraffin-embedded tumors and matched normal tissues (Fig. 1). MRP2-positive expression was observed in the tumor and matched normal tissues of $24(48 \%)$ and 7 (14\%) cases, respectively. Comparing MRP2 expression in the tumor tissues and matched normal tissues indicated significant over-
A

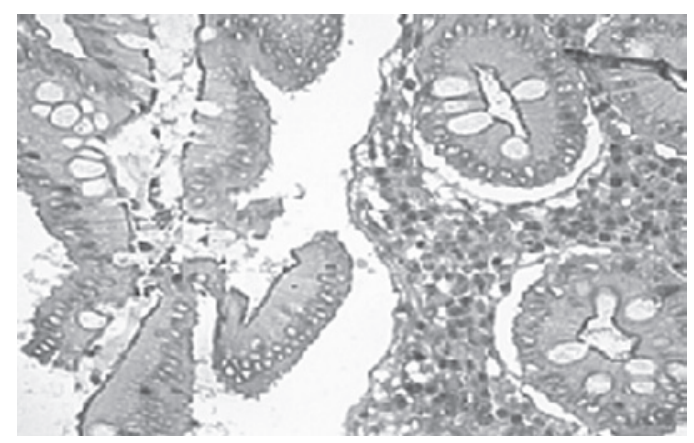

$\mathbf{B}$

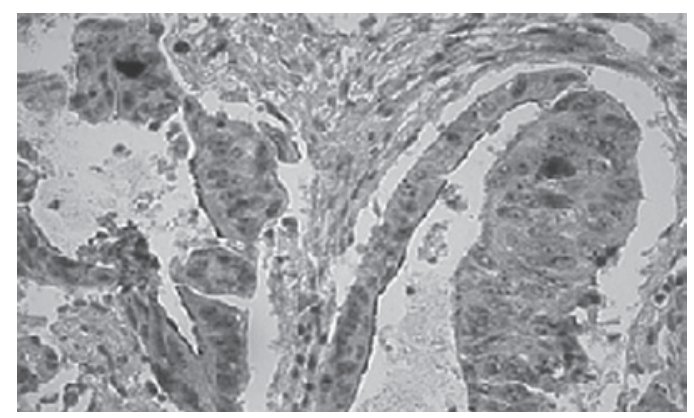

C

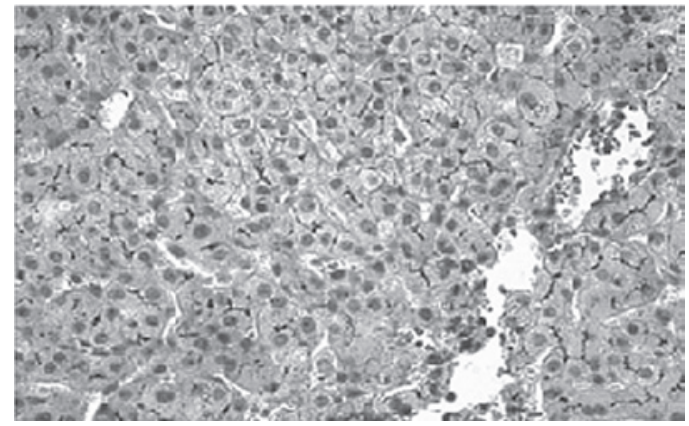

Figure 1. Immunohistochemical staining for MRP2 in paraffin-embedded tissue section using M2III-6 monoclonal antibody. (A) Strong and moderate apical MRP2 staining in glands and surface epithelium of normal mucosa. (B) Strong and weak apical (canalicular) MRP2 staining in adenocarcinoma tissue. (C) Strong MRP2 staining at the apical hepatocyte membranes. Magnification, x400. MRP2, multiple drug resistance protein 2.

expression of MRP2 in cancerous regions compared to normal mucosa $(\mathrm{P}=0.003)$.

Correlation between early relapse and clinicopathological markers. The correlation between MRP2 expression and clinicopathological parameters was also investigated. No statistical associations were observed between early relapse and gender, age, tumor size, tumor site, lymph node metastasis, cancer stage, grade or histology $(\mathrm{P}>0.05)$. However, a higher frequency of early relapse was observed among male patients (12/15 cases).

Correlation between expression of MRP2 and early relapse. When the patients were divided into groups according to their MRP2 expression, the MRP2-positive group did not demonstrate a significantly poorer response compared to the MRP2-negative group in the tumor tissue or normal mucosa $(\mathrm{P}>0.05)$. Therefore, no association between early relapse and MRP2-positive expression was identified. All 
5 cases who developed recurrences at the time of chemotherapy (during 6 months) were MRP2-positive $(\mathrm{P}=0.022)$.

Correlation between clinicopathological factors and patient survival. Variables including clinical AJCC/UICC stage, lymph node metastasis, histology, tumor size, tumor location, age, gender and positive MRP2 expression in tumor and matched normal tissues were analyzed using the Kaplan-Meier method. These parameters were not associated with the OS or DFS rate of patients $(\mathrm{P}>0.05)$, except in the cases of deep tumor invasion. Although a significant correlation between deep tumor invasion and survival time was observed, the results were not suitable for evaluation due to the small number of cases ( 2 out of 50 ) in the T4 type group. In addition, a similar trend was observed between MRP2-negative patients and a higher OS.

\section{Discussion}

Development of drug resistance and recurrence that frequently occurs in cancer therapy is a major limitation to applying adequate high doses of drugs to eradicate tumor cells. The detection of markers that predict drug resistance is of major interest in selecting the most effective medication in first-line treatment. At present, oxaliplatin is used in the treatment of CRC (11) and combining oxaliplatin to a backbone of FL was revealed to improve the adjuvant chemotherapy of colon cancer (22). However, certain patients remain unresponsive to this effective combination chemotherapy.

MRP2 mediates the export of intracellular glutathione conjugates of a number of clinically important drugs $(9,10)$. Glutathione-conjugate formation is a well-known mechanism of resistance to oxaliplatin in CRC (11). Once oxaliplatin is passively absorbed by cells, it is detoxified within the cytoplasm by forming glutathione conjugates and consequently eliminated from the cancer cells. However, in the nucleus it forms oxaliplatin-DNA adducts that contribute to cytotoxicity. A glutathione-conjugation-oxaliplatin complex was verified to be a substrate of ABC transporters (11). Additionally, MRP2 mRNA upregulation was identified to be associated with decreased formation of platinum-DNA adducts and decreased G2-arrest in the cisplatin-resistant cell line (17). Hinoshita et al (23) also observed the association between MRP2 mRNA levels and cisplatin resistance in colorectal carcinoma cells in an in vivo study.

Taking into consideration the involvement of MRP2 in the resistance of platinating agents, we aimed to evaluate its possible association with CRC recurrence in platinum-based chemotherapy employing a homogenous population. Patients were diagnosed at an age less than 78 years in stage II/III $\mathrm{CRC}$ and did not receive preoperative treatment prior to their FOLFOX-4 regimen. We anticipated that the overexpression of MRP2 may influence tumor response and induce recurrence.

When the correlation of the MRP2-positive expression to the clinicopathological markers was examined, the statistics did not reveal any significant correlation. The MRP2-positive patients did not demonstrate a significantly superior OS and DFS compared to the MRP2-negative patients in the neoplastic tissue or normal mucosa. However, a higher OS was observed in the MRP2-negative patients. In addition, when the expression of MRP2 in the colorectal carcinoma and matched normal mucosa of patients were assessed by immunohistochemical staining, the statistical result indicated significant overexpression in the tumor tissue. Therefore, the expression of MRP2 may play a role in the malignant transformation of colorectal tissues in selected patients. In a previous study by Hinoshita et al (23) using quantitative reverse-transcription polymerase chain reaction (RT-PCR), it was demonstrated that the expression of MRP2 was significantly increased in CRC tissues compared to normal mucosal tissues. Although overexpression of MRP2 was revealed to confer drug resistance in tumor cells (24), our observation did not identify any significant correlation between early CRC relapse and MRP2-positive expression in the neoplastic tissue or matched normal mucosa. Therefore, expression of MRP2 did not appear to play a prognostic role in CRC patients receiving adjuvant chemotherapy with FL and oxaliplatin.

We identified a subpopulation with MRP2-positive expression among patients who had poor CRC prognosis and demonstrated recurrence at time of chemotherapy. Although this number of patients was small, they were the only patients who demonstrated recurrence within 6 months. The findings indicated that there are other existing mechanisms that affect the results of the study. We suggest that further study of MRP2 expression with proteins that simultaneously transport MRP2 substrates is required. A study of the expression of organic anion transporting polypeptide (OATP2) along with MRP2 in platinum-based chemotherapy may be required.

The association between MRP2 expression and the clinical outcome has been demonstrated in various types of tumors (25-27), but its prognostic role in CRC remains to be clarified. Further study is required to elucidate the role of MRP2 expression in poor response patients who received platinum-based therapy.

In conclusion, we demonstrated that MRP2 was overexpressed in tumor tissues compared to normal tissues. To the best of our knowledge, this is the first study to indicate the protein expression of the ABC transporter MRP2 in tumor and normal tissue. We identified that MRP2 expression is not a predictive value of poor clinical outcome in patients with stage II/III CRC treated with FL and oxaliplatin chemotherapy.

\section{References}

1. Malekzadeh R, Bishehsari F, Mahdavinia M and Ansari R: Epidemiology and molecular genetics of colorectal cancer in Iran: a review. Arch Iran Med 12: 161-169, 2009.

2. Sadjadi A, Nouraie M, Mohagheghi MA, Mousavi-Jarrahi A, Malekzadeh R and Parkin DM: Cancer occurrence in Iran in 2002: an international perspective. Asian Pac J Cancer Prev 6: 359-363, 2005.

3. Catalano V, Baldelli AM, Giordani P and Cascinu S: Molecular markers predictive of response to chemotherapy in gastrointestinal tumors. Crit Rev Oncol Hematol 38: 93-104, 2001.

4. Beck WT: Circumvention of multidrug resistance with anti-P-glycoprotein antibodies: clinical potential or experimental artifact? J Natl Cancer Inst 87: 73-75, 1995.

5. Takano M, Yumoto R and Murakami T: Expression and function of efflux drug transporters in the intestine. Pharmacol Ther 109: 137-161, 2006.

6. Sandusky GE, Mintze KS, Pratt SE and Dantzig AH: Expression of multidrug resistance-associated protein 2 (MRP2) in normal human tissues and carcinomas using tissue microarrays. Histopathology 41: 65-74, 2002. 
7. Kerb R, Hoffmeyer S and Brinkmann U: ABC drug transporters: hereditary polymorphisms and pharmacological impact in MDR1, MRP1 and MRP2. Pharmacogenomics 2: 51-64, 2001

8. Ohashi R, Takahashi F, Cui R, et al: Interaction between CD44 and hyaluronate induces chemoresistance in non-small cell lung cancer cell. Cancer Lett 252: 225-234, 2007.

9. Taniguchi K, Wada M, Kohno K, et al: A human canalicular multispecific organic anion transporter (cMOAT) gene is overexpressed in cisplatin-resistant human cancer cell lines with decreased drug accumulation. Cancer Res 56: 4124-4129, 1996.

10. Bakos E, Evers R, Sinkó E, Váradi A, Borst P and Sarkadi B: Interactions of the human multidrug resistance proteins MRP1 and MRP2 with organic anions. Mol Pharmacol 57: 760-768, 2000.

11. Casado E, De Castro J, Belda-Iniesta C, et al: Molecular markers in colorectal cancer: genetic bases for a customised treatment. Clin Transl Oncol 9: 549-554, 2007.

12. Giacchetti S, Perpoint B, Zidani R, et al: Phase III multicenter randomized trial of oxaliplatin added to chronomodulated fluorouracil-leucovorin as first-line treatment of metastatic colorectal cancer. J Clin Oncol 18: 136-147, 2000.

13. Allen WL, Coyle VM and Johnston PG: Predicting the outcome of chemotherapy for colorectal cancer. Curr Opin Pharmacol 6 : 332-336, 2006

14. Rabik CA and Dolan ME: Molecular mechanisms of resistance and toxicity associated with platinating agents. Cancer Treat Rev 33: 9-23, 2007.

15. Zhang K, Mack P and Wong KP: Glutathione-related mechanisms in cellular resistance to anticancer drugs. Int J Oncol 12: 871-882, 1998

16. Itoh Y, Tamai M, Yokogawa K, et al: Involvement of multidrug resistance-associated protein 2 in in vivo cisplatin resistance of rat hepatoma AH66 cells. Anticancer Res 22: 1649-1653, 2002.

17. Liedert B, Materna V, Schadendorf D, Thomale J and Lage H: Overexpression of cMOAT (MRP2/ABCC2) is associated with decreased formation of platinum-DNA adducts and decreased G2-arrest in melanoma cells resistant to cisplatin. J Invest Dermatol 121: 172-176, 2003.
18. Shen K, Cui D, Sun L, Han M and Liu J: Inhibition of IGF-IR increases chemosensitivity in human colorectal cancer cells through MRP-2 promoter suppression. J Cell Biochem: Jan 24, 2012 (Epub ahead of print).

19. Sobin LH and Wittekind C (eds): International Union Against Cancer. In: TNM Classification of Malignant Tumors. 6th edition. John Wiley \& Sons, New Jersey, 2002.

20. Arts HJ, Katsaros D, de Vries EG, et al: Drug resistance associated markers P-glycoprotein, multidrug resistance associated protein 1 , multidrug resistance-associated protein 2 , and lung resistance protein as prognostic factors in ovarian carcinoma. Clin Cancer Res 5: 2798-2805, 1999.

21. Ushijima R, Takayama K,Izumi UM, et al: Immunohistochemical expression of MRP2 and clinical resistance to platinum-based chemotherapy in small cell lung cancer. Anticancer Res 27: 4351-4358, 2007.

22. André T, Boni C, Mounedji-Boudiaf L, et al: Oxaliplatin, fluorouracil, and leucovorin as adjuvant treatment for colon cancer. N Engl J Med 350: 2343-2351, 2004.

23. Hinoshita E, Uchiumi T, Taguchi K, et al: Increased expression of an ATP-binding cassette superfamily transporter, multidrug resistance protein 2 , in human colorectal carcinomas. Clin Cancer Res 6: 2401-2407, 2000.

24. Borst P, Evers R, Kool M and Wijnholds J: A family of drug transporters: the multidrug resistance-associated proteins. J Natl Cancer Inst 92: 1295-1302, 2000.

25. Yamasaki M, Makino T, Masuzawa T, et al: Role of multidrug resistance protein 2 (MRP2) in chemoresistance and clinical outcome in oesophageal squamous cell carcinoma. Br J Cancer 104: 707-713, 2011.

26. Van den Broek GB, Wildeman M, Rasch CR, et al: Molecular markers predict outcome in squamous cell carcinoma of the head and neck after concomitant cisplatin-based chemoradiation. Int J Cancer 124: 2643-2650, 2009.

27. Maciejczyk A, Jagoda E, Wysocka T, Matkowski R, Györffy B, Lage $\mathrm{H}$ and Surowiak P: ABCC2 (MRP2, cMOAT) localized in the nuclear envelope of breast carcinoma cells correlates with poor clinical outcome. Pathol Oncol Res 18: 331-342, 2012. 